\title{
CARATHÉODORY COMPLETENESS ON THE PLANE
}

\author{
BY ARMEN EDIGARIAN \\ Dedicated to the memory of Professor Józef Siciak
}

\begin{abstract}
M. A. Selby $8 \mathbf{1 0}$ and, independently, N. Sibony $\mathbf{1 1}$ proved that on the complex plane $c$-completeness is equivalent to $c$-finitely compactness. Their proofs are quite similar and are based on $[4$. We give more refined equivalent conditions and, along the way, simplify the proofs.
\end{abstract}

1. Introduction. Let $D \subset \mathbb{C}^{n}$ be a domain and let $\zeta \in \partial D$ be its boundary point. We denote by $A(D \cup\{\zeta\})$ the set of all bounded holomorphic functions on $D$ which extend continuously to $D \cup\{\zeta\}$. Following $[7]$, we say that $\zeta$ is a weak peak point for $D$ if there exists a function $f \in A(D \cup\{\zeta\})$ such that $|f|<1$ on $D$ and $f(\zeta)=1$.

Theorem 1. Let $D \subset \mathbb{C}$ be a domain and let $\zeta \in \partial D$ be its boundary point. Then the following conditions are equivalent:

(1) $\zeta$ is a weak peak point for $D$;

(2) there exists no finite Borel measure $\mu$ on D such that

$$
|f(\zeta)| \leq \int|f| d \mu \quad \text { for any } f \in A(D \cup\{\zeta\}) ;
$$

(3) we have

$$
\sum_{n=1}^{\infty} 2^{n} \gamma\left(A_{n}(\zeta, a) \backslash D\right)=+\infty
$$

2010 Mathematics Subject Classification. 30H05, 30H50.

Key words and phrases. Carathéodory distance, completeness, Melnikov's theorem, peak function.

The author was supported in part by the Polish National Science Centre (NCN) grant no. 2015/17/B/ST1/00996. 
where $A_{n}(\zeta)=\left\{z \in \mathbb{C}: \frac{1}{2^{n+1}} \leq|z-\zeta| \leq \frac{1}{2^{n}}\right\}$ and $\gamma$ is the analytic capacity (see the definition below).

The equivalency of (1) and (3) in Theorem 1 was proved by M. A. Selby (see $[9]$ ). Note that the implication (1) $\Longrightarrow(2)$ is straightforward (also in a higher dimension). The implication $(2) \Longrightarrow(1)$ in any dimension is claimed in [2]. However, the proof is based on a false version of Hahn-Banach theorem, claimed in $[\mathbf{5}$. So, we give a new proof on the complex plane. In a higher dimension, it is still an open problem whether $(2) \Longrightarrow(1)$.

Let $\mathbb{D}(\zeta, r)=\{z \in \mathbb{C}:|z-\zeta|<r\}$ denote the disk on the complex plane and let $\mathbb{D}=\mathbb{D}(0,1)$ denote the unit disk. We define the Poincaré function $p$ on $\mathbb{D}$ as

$$
p\left(\lambda_{1}, \lambda_{2}\right)=\frac{1}{2} \log \frac{1+m\left(\lambda_{1}, \lambda_{2}\right)}{1-m\left(\lambda_{1}, \lambda_{2}\right)}, \quad \lambda_{1}, \lambda_{2} \in \mathbb{D},
$$

where $m\left(\lambda_{1}, \lambda_{2}\right)=\left|\frac{\lambda_{1}-\lambda_{2}}{1-\bar{\lambda}_{1} \lambda_{2}}\right|$ is the Möbius function.

Let $D \subset \mathbb{C}^{n}, n \geq 1$, be a domain. For $z_{1}, z_{2} \in D$ put

$$
\begin{aligned}
& c_{D}\left(z_{1}, z_{2}\right)=\sup \left\{p\left(f\left(z_{1}\right), f\left(z_{2}\right)\right): f \in \mathcal{O}(D ; \mathbb{D})\right\}, \\
& c_{D}^{*}\left(z_{1}, z_{2}\right)=\sup \left\{m\left(f\left(z_{1}\right), f\left(z_{2}\right)\right): f \in \mathcal{O}(D ; \mathbb{D})\right\},
\end{aligned}
$$

where $\mathcal{O}(D ; \mathbb{D})$ denotes the set of all holomorphic mappings $D \rightarrow \mathbb{D}$. $c_{D}$ is called the Carathéodory pseudodistance for $D$ (see e.g. [6] ). In case when $c_{D}$ is indeed a distance we say that $D$ is $c$-hyperbolic. A $c$-hyperbolic domain $D$ is called $c$-complete if any $c_{D}$-Cauchy sequence $\left\{z_{\nu}\right\}_{\nu \geq 1} \subset D$ converges to a point $z_{0} \in D$ (w.r.t. Euclidean topology).

The aim of this paper is to study more carefully the completeness on the complex plane. Along the way we simplify the proofs by M. A. Selby 8 and by N. Sibony $\mathbf{1 1}$.

We say that a measurable set $F \subset \mathbb{C}$ is of positive density at a point $\zeta \in \mathbb{C}$ if

$$
\limsup _{r \rightarrow 0+} \frac{\mathcal{L}(\overline{\mathbb{D}(\zeta ; r)} \cap F)}{r^{2}}>0 .
$$

First we show the following result.

Theorem 2. Let $D \subset \mathbb{C}$ be a domain and let $\zeta \in \partial D$ be its boundary point. If $\zeta$ is not a weak peak point for $D$ then

$$
\lim _{r \rightarrow 0+} \frac{\mathcal{L}(\mathbb{D}(\zeta ; r) \cap D)}{\pi r^{2}}=1 .
$$

We have the following inverse of Theorem 2 . 
Theorem 3. Let $D \subset \mathbb{C}$ be a domain and let $\zeta \in \partial D$ be its boundary point. Assume that

$$
\lim _{r \rightarrow 0+} \frac{\mathcal{L}(\mathbb{D}(\zeta ; r) \cap D)}{\pi r^{2}}=1 .
$$

Then the following conditions are equivalent to conditions (1), (2), (3) in Theorem [1:

(4) there exists a set $A \subset D$ of positive density at $\zeta$ such that for any sequence $\left\{z_{\nu}\right\}_{n \geq 1} \subset A$ with $z_{\nu} \rightarrow \zeta$ we have $c_{D}\left(z_{0}, z_{\nu}\right) \rightarrow \infty$;

(5) there exists a set $A \subset D$ of positive density at $\zeta$ such that for any sequence $\left\{z_{\nu}\right\}_{\nu \geq 1} \subset A$ such that $z_{\nu} \rightarrow \zeta$ there follows that $\left\{z_{\nu}\right\}$ is not a $c_{D}$-Cauchy sequence.

Note that the implications $(1) \Longrightarrow(4) \Longrightarrow(5)$ are straightforward. Essentially, the main result of the paper is showing that $(5) \Longrightarrow(2)$. In case $A=\Omega$ in Theorem 3 , the result is proved in $[\mathbf{8}]$ and $[\mathbf{1 1}]$.

2. Proof of Theorem 1. Recall the definition of the analytic capacity (see e.g. Chapter VIII in $[\mathbf{3} \mid$ ). Let $\widehat{\mathbb{C}}=\mathbb{C} \cup\{\infty\}$ denote the Riemann sphere. The analytic capacity of a compact set $K$ is defined by

$$
\gamma(K)=\sup \left\{\left|f^{\prime}(\infty)\right|: f \in \mathcal{O}(\Omega),\|f\| \leq 1, f(\infty)=0\right\},
$$

where $\Omega$ is the unbounded component of $\widehat{\mathbb{C}} \backslash K$ and

$$
f^{\prime}(\infty)=\lim _{z \rightarrow \infty} z(f(z)-f(\infty)) .
$$

For any set $F \subset \mathbb{C}$ we put

$$
\gamma(F)=\sup \{\gamma(K): K \subset F \text { compact }\} .
$$

Recall also the following characterization (see Theorem VIII.4.5 in $[\mathbf{3}]$ ).

Theorem 4 (Melnikov's criterion). Let $K \subset \mathbb{C}$ be a compact set and let $\zeta \in K$. Then $\zeta$ is a peak point for $R(K)$ if and only if

$$
\sum_{n=1}^{\infty} 2^{n} \gamma\left(A_{n}(\zeta) \backslash K\right)=+\infty .
$$

Note that the implication $(1) \Longrightarrow(2)$ in Theorem 1 is immediate. Let us show the implication $(2) \Longrightarrow(3)$.

Proof of $(2) \Longrightarrow(3)$ In Theorem 1. Assume that

$$
\sum_{n=1}^{\infty} 2^{n} \gamma\left(A_{n}(\zeta) \backslash D\right)<+\infty .
$$


Using the continuity property of the analytic capacity (see the proof of Theorem 3.1 in [4]) one can show that there exists a compact set $K \subset D \cup\{\zeta\}$ such that

$$
\sum_{n=1}^{\infty} 2^{n} \gamma\left(A_{n}(\zeta) \backslash K\right)<+\infty .
$$

By Melnikov's criterion $\zeta$ is a not peak point for $R(K)$. Hence, by Bishop's characterization of peak points (see e.g. [3]) there exists a Borel probability measure $\mu$ on $K$ such that $\mu(\{\zeta\})=0$ and

$$
f(\zeta)=\int f d \mu \quad \text { for any } f \in R(K) .
$$

Note that $A(D \cup\{\zeta\}) \subset R(K)$ (see Corollary 8 below). Hence,

$$
f(\zeta)=\int f d \mu \quad \text { for any } f \in A(D \cup\{\zeta\}) .
$$

A contradiction.

3. Proof of Theorem 2. Let $\mathcal{L}$ denote the Lebesgue measure in $\mathbb{C}$. Recall the following well-known result (see e.g. [1], Lemma 1.5).

Proposition 5. Let $K \subset \mathbb{C}$ be a compact set. Then the function

$$
f(z)=\int_{K} \frac{d \mathcal{L}(\eta)}{z-\eta}
$$

is holomorphic on $\widehat{\mathbb{C}} \backslash K$, continuous on $\widehat{\mathbb{C}}$ and $f(\infty)=0$. Moreover,

$$
|f(z)| \leq \int_{K} \frac{1}{|z-\eta|} d \mathcal{L}(\eta) \leq 2 \sqrt{\pi \mathcal{L}(K)}
$$

As a corollary of Proposition 5 we get Theorem 2 (cf. Corollary VIII.4.2 in $[\mathbf{3} \mid)$.

Proof of Theorem 2. Assume that

$$
\limsup _{r \rightarrow 0+} \frac{\mathcal{L}(\overline{\mathbb{D}(\zeta ; r)} \backslash D)}{r^{2}}>0 .
$$

Choose $r_{n} \rightarrow 0+$ and $b>0$ such that $\mathcal{L}\left(K_{n}\right)>b r_{n}^{2}$, where $K_{n}=\overline{\mathbb{D}\left(\zeta ; r_{n}\right)} \backslash D$. Put

$$
g_{n}(z)=\frac{1}{\mathcal{L}\left(K_{n}\right)} \cdot(z-\zeta) \int_{K_{n}} \frac{d \mathcal{L}(\eta)}{z-\eta} .
$$

From Proposition 5 there follows that $g_{n}$ is a continuous function on $\widehat{\mathbb{C}}$, holomorphic on $\widehat{\mathbb{C}} \backslash K_{n}, g_{n}(\infty)=1$. 
Note that for any $z \in \mathbb{C}$ such that $|z-\zeta| \leq r_{n}$ we have

$$
\left|g_{n}(z)\right| \leq \frac{2 r_{n} \sqrt{\pi \mathcal{L}\left(K_{n}\right)}}{\mathcal{L}\left(K_{n}\right)} \leq 2 \sqrt{\frac{\pi}{b}} .
$$

From the maximum principle we see that the above inequality holds on the whole $\widehat{\mathbb{C}}$. Now we proceed as in the proof of Theorem VIII.4.1 in $[3]$ and get a weak peak function for $D$.

4. Proof of Theorem 3. We denote by $\mathcal{M}$ the set of all positive finite Borel measures in $\mathbb{C}$. For $\mu \in \mathcal{M}$ we define its Newton potential as

$$
M(z)=M_{\mu}(z)=\int \frac{1}{|z-\eta|} d \mu(\eta) .
$$

From the inequality (3) we have

$$
\frac{1}{\pi r^{2}} \int_{\mathbb{D}(\eta, r)}|z-\eta| \cdot M(z) d \mathcal{L}(z) \leq 2 \mu(\mathbb{C}),
$$

and, therefore, $M<\infty$ a.e. on $\mathbb{C}$. The following result, which essentially is a corollary of Fubini's theorem, shows the behaviour of the left side of (4) when $r \rightarrow 0$ (see e.g. 12, Lemma 26.16).

Proposition 6. Let $\mu \in \mathcal{M}$. For any $\eta \in \mathbb{C}$ we have

$$
\lim _{r \rightarrow 0} \frac{1}{\pi r^{2}} \int_{\mathbb{D}(\eta, r)}|z-\eta| \cdot M(z) d \mathcal{L}(z)=\mu(\{\eta\}) .
$$

In particular, if $\mu(\{\eta\})=0$, then for any $\epsilon>0$ the set

$$
\Pi(\epsilon)=\{z \in \mathbb{C}:|z-\eta| \cdot M(z)>\epsilon\}
$$

is of zero density at $\eta$, i.e.,

$$
\lim _{r \rightarrow 0} \frac{\mathcal{L}(\Pi(\epsilon) \cap \mathbb{D}(\eta, r))}{r^{2}}=0 .
$$

Recall the following approximation result (see e.g., Theorem 10.8 in Chapter VIII in $[\mathbf{3}]$ ).

Theorem 7. Let $D \subset \mathbb{C}$ be a domain and let $\zeta \in \partial D$ be its boundary point. For any $f \in H^{\infty}(D)$ there exists a sequence $\left\{f_{n}\right\}_{n \geq 1} \subset H^{\infty}(D)$ with $\left\|f_{n}\right\|_{D} \leq 17\|f\|_{D}$ such that $f_{n} \rightarrow f$ locally uniformly on $D$ and each $f_{n}$ extends holomorphically to a neighborhood of $\zeta$. Moreover, if $f$ extends continuously to $\zeta$, then $f_{n}$ tends to $f$ uniformly on $D$.

From this we get.

Corollary 8. Let $D \subset \mathbb{C}$ be a domain and let $\zeta \in \partial D$ be its boundary point. Then for any compact set $K \subset D \cup\{\zeta\}$ we have $A(D \cup\{\zeta\}) \subset R(K)$. 
The following simple observation holds true.

Proposition 9. Let $D \subset \mathbb{C}$ be a domain and let $\zeta \in \partial D$. Assume that $\mu$ is a finite Borel measure in D such that

$$
|f(\zeta)| \leq \int|f| d \mu
$$

for any $f \in A(D \cup\{\zeta\})$. Then for any $\eta \in D$ we have

$$
|f(\eta)-f(\zeta)| \leq 2\|f\|_{\infty} M(\eta)|\eta-\zeta| .
$$

In particular, for any $\eta_{1}, \eta_{2} \in D$ we have

$$
c_{D}^{*}\left(\eta_{1}, \eta_{2}\right) \leq 34\left(\left|\zeta-\eta_{1}\right| M\left(\eta_{1}\right)+\left|\zeta-\eta_{2}\right| M\left(\eta_{2}\right)\right) .
$$

Proof. Fix $\eta \in D$. Then for any $f \in A(D \cup\{\zeta\})$ we have $\widetilde{f}(z)=$ $\frac{f(z)-f(\eta)}{z-\eta} \in A(D \cup\{\zeta\})$. Then

$$
|\widetilde{f}(\zeta)| \leq \int|\widetilde{f}| d \mu .
$$

Hence,

$$
|f(\zeta)-f(\eta)| \leq|\zeta-\eta| \int\left|\frac{f(z)-f(\eta)}{z-\eta}\right| d \mu(z) \leq 2\|f\|_{\infty} M(\eta)|\eta-\zeta| .
$$

Inequality (5) follows from Theorem 7

We have the following corollary, which proofs the implication $(5) \Longrightarrow(2)$.

Corollary 10. Let $D \subset \mathbb{C}$ be a domain and let $\zeta \in \partial D$. Assume that $\mu$ is a finite Borel measure in D such that

$$
|f(\zeta)| \leq \int|f| d \mu
$$

for any $f \in A(D \cup\{\zeta\})$. Then for any measurable set $A \subset D$ of positive density at $\zeta$ there exists a c-Cauchy sequence $\left\{\eta_{n}\right\}_{n \geq 1} \subset A$ such that $\eta_{n} \rightarrow \zeta$.

Proof. If

$$
\liminf _{r \rightarrow 0} \frac{\mathcal{L}(\mathbb{D}(\zeta ; r) \cap D)}{\pi r^{2}}<1
$$

then by Theorem $2 \zeta$ is a weak peak point, which contradicts the existence of the measure $\mu$. So,

$$
\lim _{r \rightarrow 0} \frac{\mathcal{L}(\mathbb{D}(\zeta ; r) \cap D)}{\pi r^{2}}=1 .
$$

Hence,

$$
\limsup _{r \rightarrow 0} \frac{\mathcal{L}(\mathbb{D}(\zeta ; r) \cap A)}{r^{2}}>0 .
$$


Then by Proposition 6 there exists a sequence $\left\{\eta_{n}\right\}_{n \geq 1} \subset D$ with $\eta_{n} \rightarrow \zeta$ such that $\left|\zeta-\eta_{n}\right| M\left(\eta_{n}\right) \leq \frac{1}{2^{n}}$. From Theorem 9 we get the result.

\title{
References
}

1. Boivin A., Gauthier P., Holomorphic and harmonic approximation on Riemann surfaces, in the book Approximation, Complex Analysis, and Potential Theory, edited by N. Arakelian, P. M. Gauthier, and G. Sabidussi, Kluwer Academic Publishers, 2001.

2. Edigarian A., Peak points for domains in $\mathbb{C}^{n}$, Ann. Polon. Math., 114(1) (2015), 1-12.

3. Gamelin T. W., Uniform algebras, Chelsea Publishing Company, Providence, R.I., 1984.

4. Gamelin T.W., Garnett J., Distinguished homomorphisms and fiber algebras, Amer. J. Math, 92 (1970), 455-474.

5. Gogus N. G., Perkins T.L., Poletsky E. A., Non-compact versions of Edwards' theorem, Positivity, 17 (2013), 459-473.

6. Jarnicki M., Pflug P., Invariant distances and metrics in complex analysis, De Gruyter, 2nd Extended Edition, Berlin-Boston, 2013.

7. Kosiński Ł., Zwonek W., Proper holomorphic mappings vs. peak points and Shilov boundary, Ann. Polon. Math., 107 (2013), 97-108.

8. Selby M. A., On completeness with respect to the Carathéodory metric, Canad. Math. Bull., 17 (1974), 261-263.

9. Selby M. A., On maximal and complete regions, Colloq. Math., 32 (1974), 119-125.

10. Selby M. A., On completeness with respect to a Carathéodory-like metric, Colloq. Math., 39 (1978), 87-94.

11. Sibony N., Prolongement de fonctions holomorphes bornées et metrique de Carathéodory, Invent. Math., 29 (1975), 205-230.

12. Stout E. L., The theory of uniform algebras, Bogden and Quigley Publishers, Belmont, California, 1971.

Received February 15, 2019

\author{
Jagiellonian University \\ Faculty of Mathematics and Computer Science \\ Łojasiewicza 6 \\ 30-348 Kraków, Poland \\ e-mail: armen.edigarian@uj.edu.pl
}

